\title{
Electromagnetic Pulse Generated by a Horizontal Electric Dipole over a Perfect Conductor Covered with a Dielectric Layer
}

\author{
Juan Zheng ${ }^{1}$, Tong $\mathrm{He}^{1}$, Ping $\mathrm{Cao}^{1}$, Zhuhong $\mathrm{Lin}^{1}$, and Kai $\mathrm{Li}^{1}$ \\ ${ }^{1}$ Zhejiang University, College of Information Science and Electronic Engineering, China
}

\begin{abstract}
In this paper, the electromagnetic pulse due to a delta-function current excitation has been derived on the planar surface of a perfect conductor coated by a dielectric layer. The approximate expression of wave component is obtained when both the transmitting source and the receiving antennas are located on the surface of the dielectric. When the thickness of the intermediate layer is subjected to the condition of $k_{1} 1<<0.6$, this physical model is applied to the microstrip circuit. Analysis and computations of the wave components are carried out on the microstrip circuit, including the surface trapped wave vector which had been ignored in former studies. It is shown that the trapped-surface-wave terms should have been taken into consideration as the main contribution in total transient field in the far-field radiations.
\end{abstract}

\section{Introduction}

The electromagnetic fields of vertical and horizontal electric dipoles on or near the boundary of two dielectrics have been well known in terms of closed-form expressions for many years [1][2].

In early 1990's, the simple analytical formulas were derived for the electromagnetic fields generated by horizontal and vertical electric dipoles in the presence of a three-layered region. The details of the research findings are well summarized in the classic two books [3][4]. It was demonstrated that the total field on or near the air-dielectric boundary is determined primarily by lateral wave, where the amplitude of the field along the boundary is $1 / \rho^{2}$.

In the late 1990's, Wait (1998) and Mahmound (1999) wrote comments on the work by King and Sandler (1994) and considered that the surface wave, varying as $\rho^{-1 / 2}$ in the farfield region, should not be overlooked in the three-layered case [5][6].

After that, many researchers had revisited the old problem and some new progresses had been made [7-10]. Over the past two decades, the existence or nonexistence of the trappedsurface-wave term in the solution had been a controversial subject for the electromagnetic field radiated by a dipole source in the presence of three-layered region. The details of the study on the controversial subject were summarized in the book by Li [11]. However, it should be noted that the trapped-surface-wave terms were indeed underestimated in the classic book [4]. 
According to Wait, it is known that the total transient field should include the trapped surface wave as the primary wave in the far-field radiation when both the transmitting source and receiving antennas are located on the planar surface of dielectric. Therefore, the electromagnetic pulse of a vertical electric dipole in the presence of three-layered region was treated analytically in the former research by Cheng et al. [12].

Following the research line, the transient field generated by a horizontal electric dipole with delta-excitation in the presence of three-layered region has been investigated including the trapped-surface-wave term in the present study for the applications of microstrip circuit [13-17].

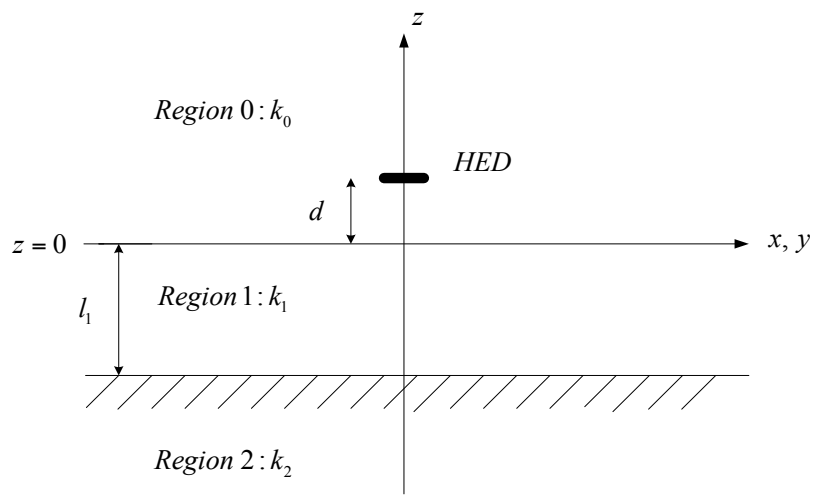

Fig.1. Horizontal electric dipole at height $d$ in air near the surface of a perfect conductor coated with a dielectric layer.

\section{Transient field of horizontal electric dipole with delta-function excitation}

\subsection{The simplified representations for the field components in frequency excitation}

Captions should be typed in 9-point Times. They should be centred above the tables and flush left beneath the figures. The geometry under consideration is illustrated in Fig. 1, where the horizontal electric dipole in the $\hat{z}$ direction is located at $(0,0, d)$. Region 0 $(z \geq 0)$ is the upper half-space filled with air characterized by the permeability $\mu_{0}$ and the relative permittivity $\varepsilon_{0}$, region 1 is occupied by the dielectric layer characterized by the permeability $\mu_{1}$, relative permittivity $\varepsilon_{1}$, and conductivity $\sigma_{1}$, and region $2(z \leq 0)$ is a perfect conductor. Then the wave numbers of the three layers are

$$
\begin{aligned}
& k_{0}=\omega \sqrt{\mu_{0} \varepsilon_{0}}=\frac{\omega}{c}, \\
& k_{1}=\omega \sqrt{\mu_{0} \varepsilon_{0} \varepsilon_{1}}=\frac{\omega \sqrt{\varepsilon_{1}}}{c}, \\
& k_{2} \rightarrow \infty .
\end{aligned}
$$

The approximated formulas were derived for the electromagnetic field in frequency domain excited by a horizontal electric dipole in the presence of three-layered region [8]. The time dependence $e^{i \omega t}$ is applied throughout the analysis. The field components can be written in the simplified form. 


$$
\begin{aligned}
E_{0 z}(\rho, \varphi, z)= & \frac{i \omega \mu_{0} I d l}{4 \pi k_{0}^{2}} \cos \varphi \cdot\left\{-\frac{\rho}{r_{1}} \cdot \frac{z-d}{r_{1}} \cdot\left(\frac{k_{0}^{2}}{r_{1}}+\frac{3 i k_{0}}{r_{1}^{2}}-\frac{3}{r_{1}^{3}}\right) e^{i k_{0} r_{1}}\right. \\
& +\frac{\rho}{r_{2}} \cdot \frac{z+d}{r_{2}} \cdot\left(\frac{k_{0}^{2}}{r_{2}}+\frac{3 i k_{0}}{r_{2}^{2}}-\frac{3}{r_{2}^{3}}\right) e^{i k_{0} r_{2}}+2 \pi k_{0}^{2} \\
& \cdot \sum_{j} \frac{\gamma_{L}^{*} \tan \gamma_{L}^{*} l \cdot e^{i \gamma_{0 E}^{*}(z+d)}}{q^{\prime}\left(\lambda_{j E}^{*}\right)} \cdot \lambda_{j E}^{* 2} \cdot H_{1}^{(1)}\left(\lambda_{j E}^{*} \rho\right) \\
& +2 i k_{0}^{3} A \sqrt{\frac{1}{\pi k_{0} \rho}} \cdot e^{i k_{0} r_{2}} \cdot\left[\sqrt{\frac{\pi}{k_{0} \rho}}+\frac{\pi}{\sqrt{2}} \cdot e^{i \frac{\pi}{4}}\right. \\
& \cdot A \cdot \exp \left[-i \frac{k_{0} \rho}{2}\left(\frac{z+d}{\rho}+i T\right)^{2}\right] \\
& \left.\cdot \operatorname{erfc}\left(\sqrt{\left.-i \frac{k_{0} \rho}{2}\left(\frac{z+d}{\rho}-i A\right)^{2}\right)}\right]\right\}
\end{aligned}
$$

In above formula, $A$ and $T$ are expressed by

$$
\begin{gathered}
A=\frac{k_{0}}{k_{1}^{2}} \sqrt{k_{1}^{2}-k_{0}^{2}} \tan \left(\sqrt{k_{1}^{2}-k_{0}^{2}} \cdot l\right), \\
T=\frac{\sqrt{k_{1}^{2}-k_{0}^{2}}}{k_{0} \tan \left(\sqrt{k_{1}^{2}-k_{0}^{2}} \cdot l\right)} .
\end{gathered}
$$

With $r_{1,2}=\sqrt{\rho^{2}+(z \mp d)^{2}}, q^{\prime}\left(\lambda_{j E}^{*}\right)$ and $p^{\prime}\left(\lambda_{j B}^{*}\right)$ are expressed as

$$
\begin{aligned}
& q^{\prime}\left(\lambda_{j E}^{*}\right)=-\frac{k_{1}^{2} \lambda_{j E}^{*}}{\gamma_{0 E}^{*}}+i \frac{k_{0}^{2} \lambda_{j E}^{*}}{\gamma_{1 E}^{*}}\left(\tan \gamma_{1 E}^{*} l+\gamma_{1 E}^{*} l \sec ^{2} \gamma_{1 E}^{*} l\right), \\
& p^{\prime}\left(\lambda_{j B}^{*}\right)=-\frac{\lambda_{j B}^{*}}{\gamma_{1 B}^{*}}+i \lambda_{j B}^{*}\left(\frac{\tan \gamma_{1 B}^{*} l}{\gamma_{0 B}^{*}}+\frac{\gamma_{0 B}^{*} l}{\gamma_{1 B}^{*}} \sec ^{2} \gamma_{1 B}^{*} l\right) .
\end{aligned}
$$

In above formulas, both $\gamma_{m E}^{*}$ and $\gamma_{m B}^{*}$ are written as

$$
\begin{array}{ll}
\gamma_{m E}^{*}=\sqrt{k_{m}^{2}-\left(\lambda_{j E}^{*}\right)^{2}} ; & m=0,1, \\
\gamma_{m B}^{*}=\sqrt{k_{m}^{2}-\left(\lambda_{j B}^{*}\right)^{2}} ; & m=0,1 .
\end{array}
$$

Where $m$ denotes the region numbers 0,1 and $j$ denotes the number of poles $(0 \sim \mathrm{n}), \lambda_{j E}^{*}$ and $\lambda_{j B}^{*}$ are the roots of the following two equations, respectively.

$$
\begin{gathered}
q(\lambda)=k_{1}^{2} \gamma_{0}-i k_{0}^{2} \gamma_{1} \tan \gamma_{1} l=0 \\
p(\lambda)=\gamma_{1}-i \gamma_{0} \tan \gamma_{1} l=0
\end{gathered}
$$

In general, the thickness of the intermediate layer satisfied to the condition of $k_{1} l \leq 0.6$, we have

$$
\tan \sqrt{k_{1}^{2}-k_{0}^{2} l} \approx \frac{w l}{c} \sqrt{\varepsilon_{1}-1}
$$

Defining $Q_{j}^{*}=\frac{\lambda_{j}^{*}}{k_{0}}$, thus we write

$$
q^{\prime}\left(\lambda_{j}^{*}\right) \approx-\frac{\omega^{2} Q_{j}^{*}}{c^{2}}\left(\frac{\varepsilon_{1}}{\sqrt{1-Q_{j}^{* 2}}}-i 2 \frac{\omega l}{c}\right)
$$

Both the formulas of the lateral wave and the surface wave can be reduced as follows 


$$
\begin{aligned}
& E_{0 z}^{l}(\rho, \phi, z)=-\frac{\omega \mu_{0}}{2 \pi} \cos \phi \cdot k_{0}^{2} l \cdot e^{i k_{0} r_{2}} \cdot\left(\frac{1}{k_{0} \rho}+k_{0} l \sqrt{\frac{\pi}{k_{0} \rho}} \cdot e^{-i p^{*}} \cdot F\left(p^{*}\right)\right), \\
& E_{0 z}^{s}(\rho, \phi, z) \cong \frac{\mu_{0} I d l}{2} \cos \phi \sqrt{\frac{2}{\pi \rho^{\prime}}} \times \sum_{j} \frac{\left(\varepsilon_{1}-Q_{j E}^{* 2}\right)\left(Q_{j E}^{*}\right)^{\frac{1}{2}} \sqrt{1-Q_{j E}^{* 2}} l}{2 c \sqrt{1-Q_{j E}^{* 2}} l \cdot(\omega-b)} \cdot i(\omega)^{\frac{5}{2}} e^{i \frac{3 \pi}{4}} e^{i Q_{j E}^{*} \omega \rho^{\prime}} .
\end{aligned}
$$

With above approximations, the electromagnetic field in frequency domain excited by a horizontal dipole can be rewritten in the following simplified forms

$$
\begin{aligned}
E_{0 z}(\rho, \phi, z)= & -\frac{i \omega \mu_{0} I d l}{4 \pi k_{0}} \cos \phi \cdot\left[\left(\frac{\rho}{r_{1}}\right)\left(\frac{z-d}{r_{1}}\right)\left(\frac{k_{0}}{r_{1}}-\frac{3 i}{r_{1}^{2}}+\frac{3 i}{k_{0} r_{1}^{3}}\right) e^{i k_{0} r_{1}}\right. \\
& \left.-\left(\frac{\rho}{r_{2}}\right)\left(\frac{z+d}{r_{2}}\right)\left(\frac{k_{0}}{r_{2}}-\frac{3 i}{r_{2}^{2}}-\frac{3 i}{k_{0} r_{2}^{3}}\right) e^{i k_{0} r_{2}}\right]+\frac{\mu_{0} I d l}{2} \cos \phi \sqrt{\frac{2}{\pi \rho^{\prime}}} \\
& \times \sum_{j} \frac{\left(\varepsilon_{1}-Q_{j E}^{* 2}\right)\left(Q_{j E}^{*}\right)^{\frac{1}{2}} \sqrt{1-Q_{j E}^{* 2}} l}{2 c \sqrt{1-Q_{j E}^{* 2}} l \cdot(\omega-b)} i(\omega)^{\frac{5}{2}} e^{i \frac{3 \pi}{4}} e^{i Q_{j E}^{*} \omega \rho^{\prime}} \\
& +\frac{\omega \mu_{0} I d l}{4 \pi} \cos \phi \cdot k_{0}^{2} l \cdot e^{i k_{0} r_{2}} \\
& \cdot\left[\frac{1}{k_{0} \rho}+k_{0} l \sqrt{\frac{\pi}{k_{0} \rho}} \cdot e^{-i p^{*}} \cdot F\left(p^{*}\right)\right]
\end{aligned}
$$

With $\rho^{\prime}=\frac{\rho}{c}, p^{*}$ can be written as

$$
p^{*}=-\frac{\omega^{3} l^{2} \rho^{\prime}}{2 c^{2}} \cdot \frac{\left(\varepsilon_{1}-1\right)^{2}}{\varepsilon_{1}^{2}}
$$

The Fresnel function $F\left(p^{*}\right)$ is defined by

$$
F\left(p^{*}\right)=\frac{1}{2}(1+i)-\int_{0}^{p^{*}} \frac{e^{i t}}{\sqrt{2 \pi t}} d t
$$

\subsection{Approximately formulas for transient field}

Obviously, the analytical solution of the transient electromagnetic field can be obtained from the inverse Fourier transformation of the frequency-domain solution. The three timedependent field components are described as follows

Where

$$
\begin{aligned}
E_{0 z}(\rho, 0 ; t)= & \frac{1}{\pi} \operatorname{Re} \int_{0}^{\infty} E_{0 z}(\rho, 0 ; t) e^{-i \omega t} d \omega \\
= & \frac{\mu_{0}}{2 \pi c} \cos \phi \sqrt{\frac{1}{2 \pi \rho^{\prime}}} \cdot \sum_{j} \frac{\left(\varepsilon_{1}-Q_{j E}^{* 2}\right)\left(Q_{j E}^{*}\right)^{\frac{1}{2}} \sqrt{Q_{j}^{* 2}-1} l}{\sqrt{1-Q_{j E}^{* 2}} l} I_{1} \\
& -\frac{\mu_{0}}{2 \pi} \cos \phi\left(\frac{l}{c^{2} \rho^{\prime 2}} I_{2}+\frac{\varepsilon_{1}^{2}}{c\left(\varepsilon_{1}-1\right)^{2}} \sqrt{\frac{\pi}{\rho^{\prime}}} I_{3}\right)
\end{aligned}
$$




$$
\begin{aligned}
& I_{1}=\frac{1}{\pi} \operatorname{Re} \int_{0}^{\infty} e^{i \frac{3 \pi}{4}} \frac{i \omega^{\frac{5}{2}}}{\omega-b} e^{i \omega Q_{j}^{*} \rho^{\prime}} e^{-i \omega t} d \omega, \\
& I_{2}=\frac{1}{\pi} \operatorname{Re} \int_{0}^{\infty}(-i \omega)^{2} e^{-i \omega\left(t-\rho^{\prime}\right)} d \omega, \\
& I_{3}=\frac{1}{\pi} \operatorname{Re} \int_{0}^{\infty} \omega^{\frac{3}{2}} \varepsilon^{* 2} e^{-i p^{*}} F\left(p^{*}\right) e^{-i \omega\left(t-\rho^{\prime}\right)} d \omega .
\end{aligned}
$$

It is noted that the integrals $I_{1}$ and $I_{3}$ corresponds to the trapped surface wave and the lateral wave. Under the condition of $k_{0} \rho \geq \frac{8}{\left(k_{0} l\right)^{2}}$, we have

$$
e^{-i p^{*}} F\left(p^{*}\right) \approx \sqrt{\frac{k_{0} \rho}{\pi}} \frac{1}{k_{0}^{2} \varepsilon^{*}}\left(\frac{i k_{0}}{\rho}+\frac{1}{\rho^{2} \varepsilon^{* 2}}\right)
$$

The above three integrals $I_{1}, I_{2}$, and $I_{3}$ can be approximated in the following forms

$$
\begin{aligned}
I_{1}= & \sqrt{\frac{1}{\pi}}\left\{-b^{\frac{5}{2}}\left[C_{2} \cos \left[b\left(t-Q_{j}^{*} \rho^{\prime}\right)\right]+S_{2} \sin \left[b\left(t-Q_{j}^{*} \rho^{\prime}\right)\right]\right]\right. \\
& \left.+\frac{b}{2}\left(t-Q_{j}^{*} \rho^{\prime}\right)^{-\frac{3}{2}}\right\} U\left(t-Q_{j}^{*} \rho^{\prime}\right), \\
I_{2}= & \delta^{\prime \prime}\left(t-\rho^{\prime}\right), \\
I_{3}= & -\sqrt{\frac{1}{\pi \rho^{\prime}}} \frac{\left(\varepsilon_{1}-1\right) l}{c \varepsilon_{1}} \delta^{\prime \prime}\left(t-\rho^{\prime}\right)+\sqrt{\frac{1}{\pi \rho^{\prime}}} \frac{c \varepsilon_{1}}{\left(\varepsilon_{1}-1\right) \rho^{\prime} l} U\left(t-\rho^{\prime}\right) .
\end{aligned}
$$

The Fresnel cosine integral $C_{2}$ and sine integral $S_{2}$ are written as

$$
\begin{gathered}
C_{2}=\int_{\substack{0 \\
b\left(t-Q_{j}^{*} \rho^{\prime}\right)}}^{b\left(t-Q_{j}^{*} \rho^{\prime}\right)} \frac{\cos t}{\sqrt{t}} d t, \\
S_{2}=\int_{0}^{\sin t} \frac{\sin }{\sqrt{t}} d t .
\end{gathered}
$$

With $b=\frac{c \varepsilon_{1}}{2 l \sqrt{Q_{j}^{* 2}-1}}$, the Heaviside unit step function $U(x)$ is defined by

$$
U(x)= \begin{cases}0 & x<0 \\ 1 & x \geq 0\end{cases}
$$

With the substitutions of (16) into (13), the approximated formulas of the timedependent field components can be derived readily. The component $E_{0 z}$ can be expressed in the following forms

$$
\begin{aligned}
E_{0 z}(\rho, 0 ; t)= & -\frac{\mu_{0}}{2 \pi c} \cos \phi \cdot\left(\frac{1}{\rho^{\prime}} \delta^{\prime}\left(t-\rho^{\prime}\right)-\frac{1}{\rho^{\prime 2}} \delta\left(t-\rho^{\prime}\right)+\frac{1}{\rho^{\prime 3}} U\left(t-\rho^{\prime}\right)\right) \\
& +\frac{\mu_{0}}{2 \pi c} \cos \phi \sqrt{\frac{1}{2 \pi \rho^{\prime}}} \cdot \sum_{j} \frac{\left(\varepsilon_{1}-Q_{j E}^{* 2}\right)\left(Q_{j E}^{*}\right)^{\frac{1}{2}} l}{\sqrt{1-Q_{j E}^{* 2}} l} \cdot \frac{\varepsilon_{1}-1}{2 \varepsilon_{1}} \sqrt{\frac{1}{\pi}} \\
& \cdot\left\{-b^{\frac{5}{2}}\left[C_{2} \cos \left[b\left(t-Q_{j}^{*} \rho^{\prime}\right)\right]+S_{2} \sin \left[b\left(t-Q_{j}^{*} \rho^{\prime}\right)\right]\right]\right. \\
& \left.-\frac{b}{2}\left(t-Q_{j}^{*} \rho^{\prime}\right)^{-\frac{3}{2}}-\sqrt{\pi} \cdot b^{\frac{5}{2}} \cdot \cos \left[b\left(t-Q_{j}^{*} \rho^{\prime}\right)+\frac{3 \pi}{4}\right]\right\} \\
& \cdot U\left(t-Q_{j}^{*} \rho^{\prime}\right)+\frac{\mu_{0}}{4 \pi} \cos \phi \frac{\varepsilon_{1}^{2}}{c\left(\varepsilon_{1}-1\right)^{2}}\left[\frac{1}{\rho^{\prime 2}} \frac{c \varepsilon_{1}}{l\left(c \varepsilon_{1}-1\right)} U\left(t-\rho^{\prime}\right)\right]
\end{aligned}
$$

With similar procedures, the rest five components can be obtained readily. 


\section{Computations and discussions}

In practical applications, the radiation of a horizontal electric dipole in the three-layered region is useful in the microstrip circuit. However, it is difficult for microstrip circuits to meet the far-field approximation, since the Fresnel integral has the above approximation only when the propagation distance $\rho$ is greater than the Sommerfeld distance. For example, assume the thickness of the intermediate medium layer of the microstrip circuit is $l=0.1 \mathrm{~cm}$, the operating frequency $f \sim 0.8 \mathrm{GHz}$. Hence,

$$
\begin{aligned}
& k_{0}=\frac{2 \pi f}{c}=1.6 \pi \times \frac{10^{9}}{3 \times 10^{8}}, \\
& k_{0} l=0.01675 .
\end{aligned}
$$

The far zone condition is

$$
\rho \geq \frac{8}{k_{0}^{3} l^{2}}=445.5 \mathrm{~m}
$$

The size of the circuit device usually cannot meet the above conditions. It can be seen that the size of the media material is particularly important, generally, if it satisfies to

$$
k_{0} \rho<<\frac{1}{k_{0}^{2} l^{2}}
$$

The electromagnetic field in the air and the dielectric layer includes only the direct wave and the ideal reflected wave. Therefore, the expressions for each component of the horizontal electric dipole can be approximated as follows:

$$
\frac{k_{1}^{2}}{k_{0}^{2}} E_{1 z^{\prime}}\left(\rho, \varphi^{\prime}, 0\right)=E_{0 z^{\prime}}\left(\rho, \varphi^{\prime}, 0\right) \sim \frac{i \omega \mu_{0}}{2 \pi k_{0}}\left(k_{0} l\right) \cos \varphi^{\prime} \cdot e^{i k_{0} \rho}\left(\frac{i k_{0}}{\rho}-\frac{1}{\rho^{2}}\right)
$$

The above are the expressions of the electromagnetic field in the near zone which have a wider range of applications compared to that in far zone.

According to the previous results, the transient components of a horizontal electric dipole include the direct wave, the ideal reflected wave, the lateral wave and the surface wave. Among these terms, the lateral wave and the trapped surface wave of a vertical electric dipole converge with $\rho^{-2}$ and $\rho^{-1 / 2}$, respectively, and will not be discussed here. In this part, some numerical results of the electromagnetic field excited by a horizontal electric dipole will be given, where the variations of the distance and the dielectric parameters are considered as the main influence, the excitation source is $\delta(t)$.

With $\varepsilon_{1}=2.85$, the magnitudes of the component $E_{2 z}$ and $B_{2 \varphi}$ due to a horizontal electric dipole on the surface of a dielectric-coated conductor are computed at $\rho=15,20$, $25,30 \mathrm{~km}$, respectively and shown in Fig. 2. and Fig. 3.

With $\varepsilon_{1}=2,2.65,2.85$, and 3.65 , the magnitudes of the trapped-surface-wave terms $E_{2 z}^{S}$ in (19) are computed at $\rho=300 \mathrm{~km}$ and the result are shown in Fig. 4. Obviously, it is seen that the trapped-surface-wave terms are contributed by the poles $\lambda_{j}^{*}$, which are determined by both the thickness of the dielectric layer and the dielectric parameters. In another word, the trapped-surface-wave terms can be computed readily when the poles $\lambda_{j}^{*}$ are determined. 


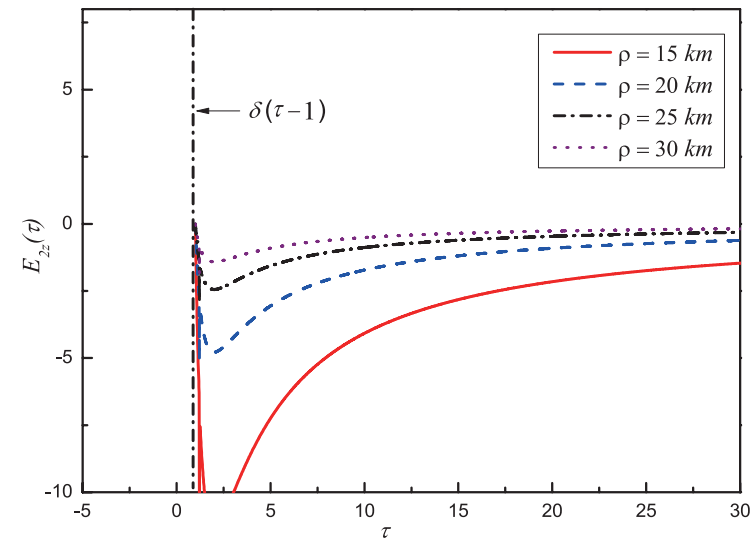

Fig.2. Magnitudes of $E_{2 z}$ due to a horizontal electric dipole with delta-function excitation. $\varepsilon_{1}=2.85$.

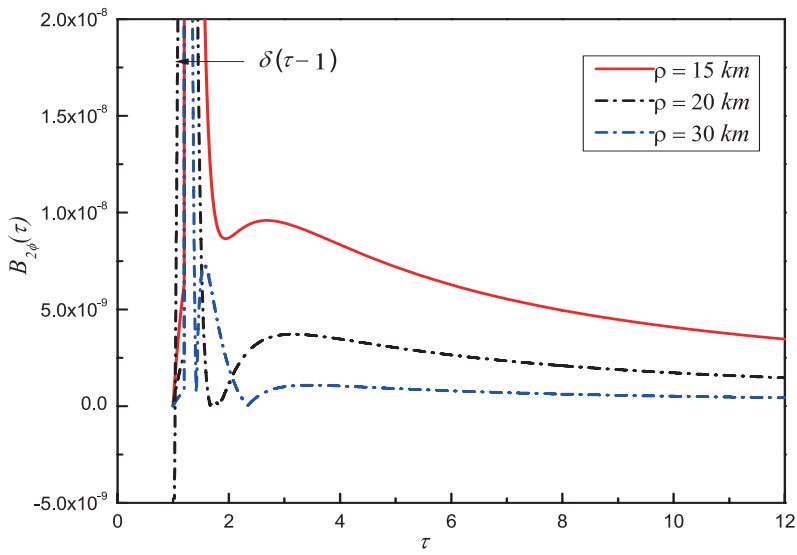

Fig.3. Magnitudes of $B_{2 \varphi}$ due to a horizontal electric dipole with delta-function excitation. $\varepsilon_{1}=2.85$.
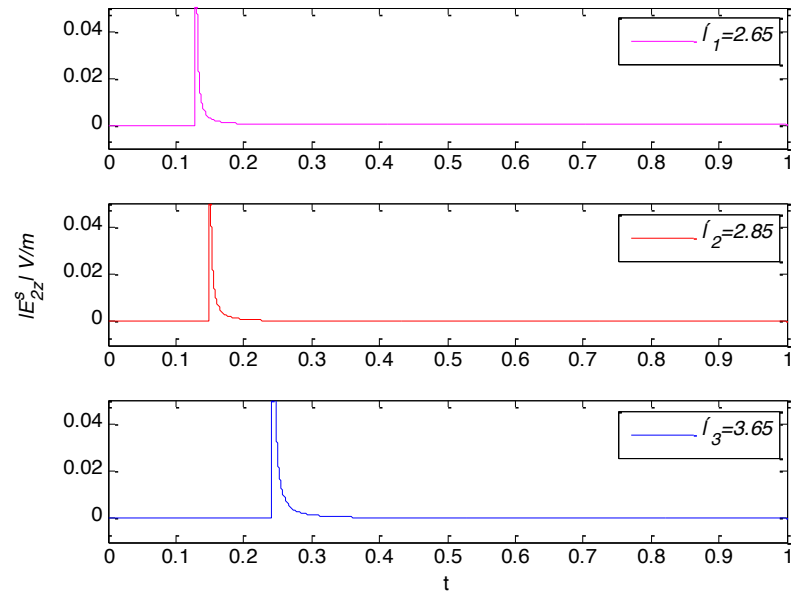

Fig.4. Magnitude of the trapped-surface-wave term $E_{2 z}^{S}(\rho, t)$ due to a horizontal electric dipole with delta-function excitation $\rho=300 \mathrm{~km}$ and $\varepsilon_{1}=2.65,2.85$ and 3.65 , respectively. 


\section{Conclusions}

In this paper, the transient electromagnetic field excited by a horizontal electric dipole with delta-function on the surface of a dielectric-coated conductor has been studied analytically. At different distances the time-dependent vertical electric field is computed and discussed, respectively. It is shown that the trapped-surface-wave terms should not be neglected in the total transient field when both the horizontal electric dipole and the observation point are on or near the surface of the dielectric-coated earth. This physical model is applied to the microstrip circuit for far-field radiations.

\section{References}

1. A. Zenneck, Annalen Physik (Leipzig), 23, 846-866 (1992)

2. A. Sommerfeld, Annalen Physik (Leipzig), 28, 665-736 (1909)

3. J. R.Wait, Pergamon Press, New York (1970)

4. R. W. P. King, M. Owens, and T. T. Wu, Springer-Verlag, New York (1992)

5. J. R. Wait, Radio Science, 33, 251-253 (1998)

6. S. F. Mahmoud, IEEE Transactions on Antennas and Propagation, 46, 1745-1946 (1999)

7. R. W. P. King and S. S. Sheldon, Radio Science, 29, pp. 97-113 (1994)

8. R. E. Collin, IEEE Transactions on Antennas and Propagation, 52, 3133-3137 (2004)

9. R. E. Collin, IEEE Antennas Propagation Maginze, 46, 64-79 (2004)

10. H.-Q. Zhang, W.-Y. Pan, K. Li, and K.-X. Shen, Progress in Electromagnetics Research, 163-186 (2005)

11. K. Li, ZJU Press, Springer (2009)

12. D. Cheng, T. T. Gu, P. Cao, T. He, and K. Li, International Journal of Antennas \& Propagation, 1-7 (2015)

13. B. F. Dong, SWJTU (2016)

14. G. Q. Zheng and B. H. Sun, The National Natural Science Fund (2017)

15. H. W. Sun, Applied Mechanics and Materials, 462-463 (2014)

16. E. A. Hajlaoui, Circuit World, 56-62 (2017)

17. S. Majidifar and G. Karimi, Ace Elsevier Journal (2016) 